\title{
Analysis of the behavior of OpenFOAM solvers for 3D problem of supersonic flow around a cone at an angle of attack
}

\author{
A.E. Bondarev, A.E. Kuvshinnikov \\ bond@keldysh.ru|kuvsh90@yandex.com \\ Keldysh Institute of Applied Mathematics RAS, Moscow, Russia;
}

\begin{abstract}
This paper is devoted to comparative analysis of numerical methods accuracy. Comparative estimation of accuracy is performed for numerical methods presented as solvers integrated to open sotware package OpenFOAM. Three different OpenFOAM solvers are selected to numerically solve the problem of supersonic flow around a cone. The angle of attack, cone half-angle and Mach number were varied in the selected ranges with a certain step. This approach is implemented using a generalized computational experiment that allows, based on parallel technologies, the simultaneous solution of the same basic problem with different input parameters. A number of test calculations were carried out. The deviation fields of gas-dynamic quantities for all solvers are analyzed. The construction of a generalized computational experiment made it possible to compare the accuracy of the considered solvers not only for one, separately taken problem, but for a class of problems specified by the ranges of variation of the determining parameters. Such an assessment of accuracy can be very useful for users of the software package when choosing a solver. Also, the results obtained can be useful for solver developers.
\end{abstract}

Keywords: comparative analysis of accuracy, compressible flow, supersonic flow, OpenFOAM.

\section{Introduction}

Currently, there are many software packages that solve the problems of flowing around elongated bodies of rotation. The researcher may ask a question: which package is best suited for such calculations. In [1, 2], it was proposed to recreate at a modern level the technology developed in the 80s at the Keldysh Institute of Applied Mathematics by A.E. Bondarev and V.A. Cherkashin under the leadership of A.V. Zabrodin. The essence of this technology is that the drag coefficient $\mathrm{Cx}$ is considered as the sum of the other three coefficients: $\mathrm{Cp}, \mathrm{Cf}$ and $\mathrm{Cd}$. This approach was widely used in the problems of mass industrial analysis of the aerodynamic properties of elongated bodies of rotation and turned out to be very effective [3].

To calculate the aerodynamic characteristics of the inviscid flow past elongated bodies of rotation, the OpenFOAM software package was used [4]. This is a free software product designed to solve the problems of hydro and gas dynamics. It is used in many fields of science and technology, both in commercial and in academic organizations. OpenFOAM contains a number of solvers with various computational properties.

It is necessary to clarify that some solvers were previously created by the developers of the OpenFOAM package [5-7], but users can create their own solvers [8]. This work is devoted to a comparative analysis of the accuracy of a number of solvers using the example of the problem of supersonic flow around a cone at an angle of attack.

Similarly to [1, 2], tabular solutions [9] were used as a reference. These tables were obtained using finitedifference methods in a wide range of Mach numbers and cone half-angle with a change in the angle of attack.

In $[1,2]$, the case of a flow near a cone at a zero angle of attack was considered. The purpose of this article is to solve a more general problem, namely, to find a flow around a cone when the angle of attack changes. The problem is solved in three-dimensional space of variable determining parameters, where the Mach number, the halfangle of the cone, and the angle of attack are considered as the determining parameters. Thus, we obtain a numerical solution for the class of problems, where the class is given by the ranges of variation of the determining parameters.

It should be noted that comparisons of solvers were also made in [10-12]. However, these comparisons were made using other examples and do not give clear recommendations on choosing a solver for the class of problems considered.

\section{Materials and method}

The statement of the problem is presented in full accordance with [9], which considers the results of an inviscid flow around cones with different half-angles of cones and angles of attack for different Mach numbers.

We study the flow around an elongated body of rotation, placed in a uniform supersonic ideal gas flow at an angle of attack $\alpha=0^{\circ}, 5^{\circ}, 10^{\circ}$ with a Mach number M $=3,5$. The body under investigation is a cone with a halfangle of $\beta=10^{\circ}, 15^{\circ}, 20^{\circ}$. The conditions of the incoming stream at the input are indicated by the index " $\infty$ ", and at the output, by the index $\xi$, since the solution is self-similar and depends on the dimensionless variable. The flow scheme is shown in Fig. 1.

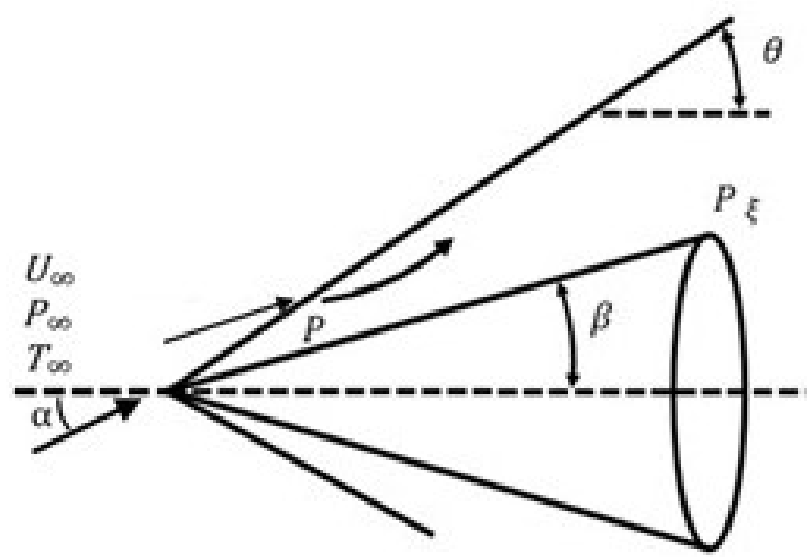

Fig. 1. Flow scheme

For calculation, the Euler system of equations is used. The system is supplemented by the ideal gas equation of state. 


\section{Compared solvers}

For comparison, 3 solvers are selected from the OpenFOAM software package:

rhoCentralFoam - is based on a central-upwind scheme which is a combination of central difference and upwind schemes $[5,6]$.

sonicFoam - is based on the PISO algorithm (Pressure Implicit with Splitting of Operator) [7].

pisoCentralFoam - is a combination of a centralupwind scheme with the PISO algorithm [8]. The pisoCentralFoam solver is not included in the standard set of solvers; it was created at the Ivannikov Institute for System Programming of the RAS.

Calculations for all solvers were carried out using the OpenFOAM version 2.3.0.

\section{Computations and results}

\section{Mesh generation, initial and boundary conditions}

Fig. 2 shows the computational domain. The setting of the boundary conditions is presented in Table 1. The parameters of the incoming flow are set on the left border, denoted as "inlet". The number of grid cells is 336000 .

The convergence study was carried out similarly to the statement of the problem in [2] and showed a satisfactory result.

\begin{tabular}{|c|c|c|c|}
\multicolumn{4}{c}{ Table 1. Boundary conditions } \\
\hline Boundary & $\mathrm{P}$ & $\mathrm{T}$ & $\mathrm{U}$ \\
\hline inlet & 101325 & 300 & $3,5 \mathrm{Mach}$ \\
\hline outlet & zeroGradient & zeroGradient & zeroGradient \\
\hline top & zeroGradient & zeroGradient & zeroGradient \\
\hline bottom & zeroGradient & zeroGradient & zeroGradient \\
\hline cone & zeroGradient & zeroGradient & slip \\
\hline front & zeroGradient & zeroGradient & zeroGradient \\
\hline back & zeroGradient & zeroGradient & zeroGradient \\
\hline
\end{tabular}

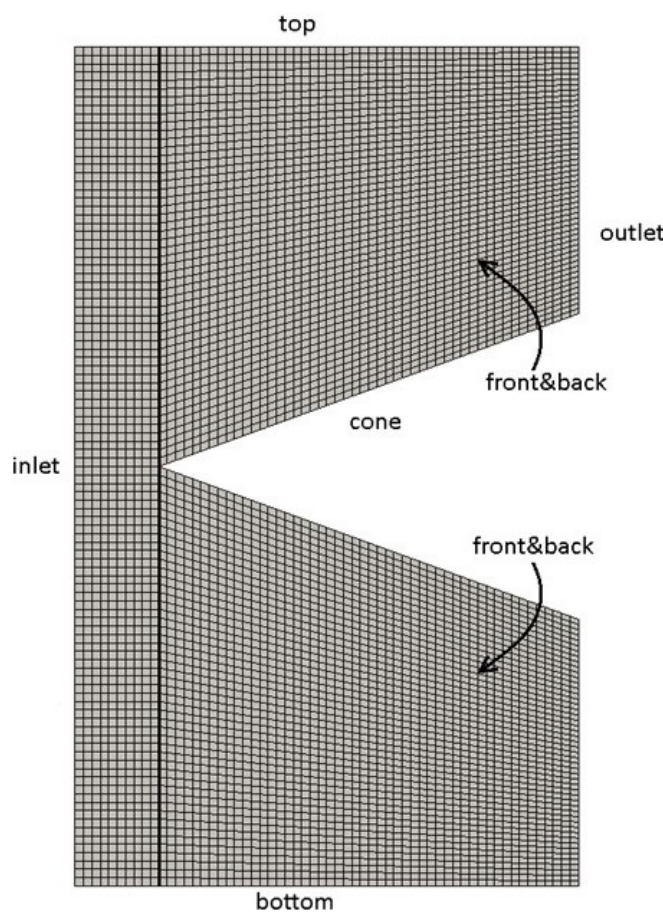

Fig. 2. Computational domain

\section{Choosing solvers parameters for unification}

In the OpenFOAM package, there are two options for approximating differential operators: directly in the solver's code or using the fvSchemes and fvSolution configuration files. To make the comparison correct, we used the same parameters where it was possible, proceeding in the same way as in $[5,6]$. In the file fvSchemes: ddtSchemes - Euler, gradSchemes - Gauss linear, divSchemes - Gauss linear, laplacianSchemes Gauss linear corrected, interpolationSchemes - vanLeer. In the file fvSolution: solver - smoothSolver, smoother symGaussSeidel, tolerance - 1e-09, nCorrectors - 2, nNonOrthogonalCorrectors - 1 .

\section{Flow calculation}

Fig. 3 shows the steady-state solution for the pressure field obtained by interpolating the tabular solution from [9], cone half-angle $\beta=20^{\circ}$, angle of attack $\alpha=10^{\circ}$, Mach number $\mathrm{M}=3$.

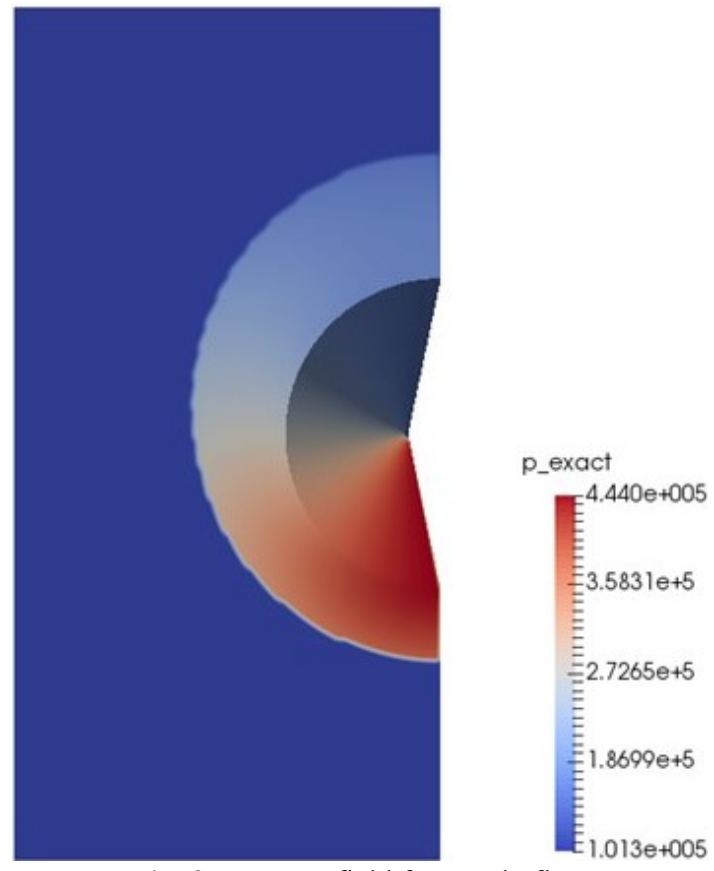

Fig. 3. Pressure field for steady flow

Tables from 2 to 7 show the result of calculation in the form of an analog of the $\mathrm{L}_{2}$ norm.

$$
\sqrt{\sum_{m}\left|y_{m}-y_{m}^{\text {exact }}\right|^{2} V_{m}} / \sqrt{\sum_{m}\left|y_{m}^{\text {exact }}\right|^{2} V_{m}}
$$

where $y_{m}$ is the pressure $p$ in the cell, $V_{m}$ is the cell volume for the cone half-angle $\beta=10^{\circ}, 15^{\circ}, 20^{\circ}$ in steps of $5^{\circ}$ and the Mach numbers $M=2-7$. The minimum values are highlighted in bold. The values of $y_{m}{ }^{\text {exact }}$ are obtained by interpolating table values from [9] into grid cells. It should be noted that the authors of the tables [9] indicate the admissibility of interpolation for all parameters and table values.

Next, we present several graphical representations of tables 2-7.

Further we will use abbreviations for solvers: rCF (rhoCentralFoam), pCF (pisoCentralFoam), $\mathrm{sF}$ (sonicFoam), QGDF (QGDFoam). 
Table 2. Deviation from the exact solution, $\mathrm{U}=3 \mathrm{M}, \beta=10^{\circ}$

\begin{tabular}{|c|c|c|c|}
\hline \multirow{2}{*}{$\begin{array}{c}\text { Angle of } \\
\text { attack }\end{array}$} & \multicolumn{3}{|c|}{ Pressure } \\
\cline { 2 - 4 } & $\mathrm{rCF}$ & $\mathrm{pCF}$ & $\mathrm{sF}$ \\
\hline 0 & $\mathbf{0 . 0 2 6 1 7 4}$ & 0.032426 & 0.046394 \\
\hline 5 & $\mathbf{0 . 0 3 0 6 3 6}$ & 0.037702 & 0.067424 \\
\hline
\end{tabular}

Table 3. Deviation from the exact solution, $U=3 \mathrm{M}, \beta=15^{\circ}$

\begin{tabular}{|c|c|c|c|}
\hline \multirow{2}{*}{$\begin{array}{c}\text { Angle of } \\
\text { attack }\end{array}$} & \multicolumn{3}{|c|}{ Pressure } \\
\cline { 2 - 4 } & $\mathrm{rCF}$ & $\mathrm{pCF}$ & $\mathrm{sF}$ \\
\hline 0 & $\mathbf{0 . 0 4 6 4 9 0}$ & 0.058198 & 0.091404 \\
\hline 5 & $\mathbf{0 . 0 5 0 2 9 8}$ & 0.060187 & 0.116237 \\
\hline 10 & $\mathbf{0 . 0 6 0 5 1 9}$ & 0.069622 & 0.145829 \\
\hline
\end{tabular}

Table 4. Deviation from the exact solution, $\mathrm{U}=3 \mathrm{M}, \beta=20^{\circ}$

\begin{tabular}{|c|c|c|c|}
\hline \multirow{2}{*}{$\begin{array}{c}\text { Angle of } \\
\text { attack }\end{array}$} & \multicolumn{3}{|c|}{ Pressure } \\
\cline { 2 - 4 } & rCF & pCF & sF \\
\hline 0 & $\mathbf{0 . 0 6 0 6 1 4}$ & 0.069128 & 0.123338 \\
\hline 5 & $\mathbf{0 . 0 6 5 3 7 3}$ & 0.075543 & 0.149785 \\
\hline 10 & $\mathbf{0 . 0 7 2 6 7 3}$ & 0.081022 & 0.172609 \\
\hline
\end{tabular}

Table 5. Deviation from the exact solution, $\mathrm{U}=5 \mathrm{M}, \beta=10^{\circ}$

\begin{tabular}{|c|c|c|c|}
\hline \multirow{2}{*}{$\begin{array}{c}\text { Angle of } \\
\text { attack }\end{array}$} & \multicolumn{3}{|c|}{ Pressure } \\
\cline { 2 - 4 } & rCF & pCF & sF \\
\hline 0 & $\mathbf{0 . 0 7 7 4 7 3}$ & 0.092200 & 0.153549 \\
\hline 5 & $\mathbf{0 . 0 9 2 1 4 9}$ & 0.100937 & 0.195634 \\
\hline
\end{tabular}

Table 6. Deviation from the exact solution, $U=5 \mathrm{M}, \beta=15^{\circ}$

\begin{tabular}{|c|c|c|c|}
\hline \multirow{2}{*}{$\begin{array}{c}\text { Angle of } \\
\text { attack }\end{array}$} & \multicolumn{3}{|c|}{ Pressure } \\
\cline { 2 - 4 } & rCF & pCF & sF \\
\hline 0 & $\mathbf{0 . 1 1 3 7 9 1}$ & 0.119559 & 0.217991 \\
\hline 5 & $\mathbf{0 . 1 2 5 8 7 3}$ & 0.130514 & 0.255976 \\
\hline 10 & $\mathbf{0 . 1 4 6 4 9 6}$ & 0.150704 & 0.294785 \\
\hline
\end{tabular}

Table 7. Deviation from the exact solution, $\mathrm{U}=5 \mathrm{M}, \beta=20^{\circ}$

\begin{tabular}{|c|c|c|c|}
\hline \multirow{2}{*}{$\begin{array}{c}\text { Angle of } \\
\text { attack }\end{array}$} & \multicolumn{3}{|c|}{ Pressure } \\
\cline { 2 - 4 } & $\mathrm{rCF}$ & $\mathrm{pCF}$ & $\mathrm{sF}$ \\
\hline 0 & 0.146504 & $\mathbf{0 . 1 3 7 9 5 4}$ & 0.265875 \\
\hline 5 & 0.152228 & $\mathbf{0 . 1 5 1 6 9 8}$ & 0.289013 \\
\hline 10 & 0.169685 & $\mathbf{0 . 1 6 2 0 0 5}$ & 0.338927 \\
\hline
\end{tabular}

Fig. 4 shows the dependence of the deviation from the exact solution in an analog of the norm $\mathrm{L}_{2}$ for pressure for the cone half-angle $\beta=20^{\circ}$ and the incoming flow Mach number $M=3$ with variation of the angle of attack $\alpha$ and solvers (Table IV). One can notice an increase in deviation with increasing angle of attack. The increase in the deviation with increasing Mach number is also clearly visible. Similar ratios are observed in other tables.

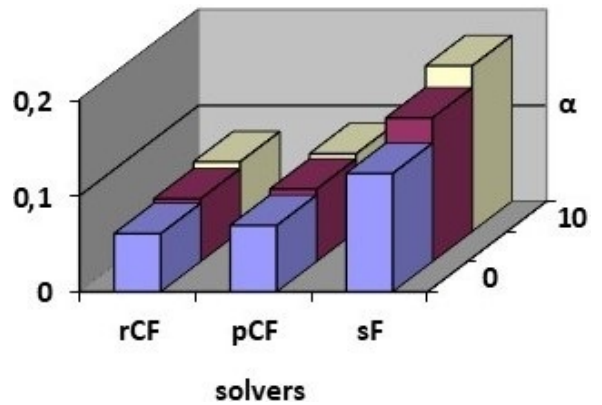

Fig. 4. Change in deviation from the exact solution for pressure depending on the solver and the angle of attack for the cone half-angle $20^{\circ}$ and Mach number 3

Fig. 5 shows the dependence of the deviation from the exact solution in an analog of the norm $\mathrm{L}_{2}$ for pressure for the angle of attack $\alpha=5^{\circ}$ and the Mach number $M=3$ with variation of the choice of solver and the angle of the halfcone of the cone. Here, with an increase in the half-angle of the cone, the deviation from the exact solution also increases.

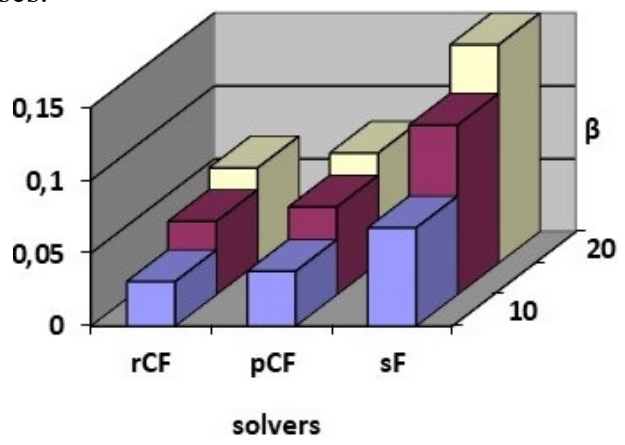

Fig. 5. Change in deviation from the exact solution for pressure depending on the solver and cone half-angle for the angle of attack $5^{\circ}$ and Mach number

\section{Conclusion}

The results show that the rhoCentralFoam solver has a minimum error rate for the pressure field. The pisoCentralFoam solver is second in accuracy.

Solver sonicFoam has oscillations at the front of the shock wave. Such oscillations are amplified with an increase in the angle of attack and the cone half-angle. Thus, the error rates of this solver are maximum among all compared solvers. Thus, it can be argued that the solvers rhoCentralFoam and pisoCentrlFoam provide the best accuracy for the class of problems and can be used in the construction of computational technology for calculating the flow for elongated bodies of rotation.

\section{Acknowledgments}

This work was supported by RFBR grants 19-0100402 and 20-01-00358.

\section{References}

[1] Bondarev A.E., Kuvshinnikov A.E. Comparative study of the accuracy for OpenFOAM solvers. - 2017 Ivannikov ISPRAS Open Conference (ISPRAS), Moscow, pp. 132-136, 2017.

[2] Bondarev A.E., Kuvshinnikov A.E. Analysis of the accuracy of OpenFOAM solvers for the problem of supersonic flow around a cone. - In: Shi Y. et al. (eds) Computational Science - ICCS 2018. ICCS 2018. Lecture Notes in Computer Science, vol. 10862. Springer, Cham, 2018.

[3] Krasil'shchikov A.P., Gur'yashkin L.P. Eksperimental'nye issledovaniya tel vrashcheniya $\mathrm{v}$ giperzvukovykh potokakh. [Experimental investigations of bodies of rotation in hypersonic flows]. - Moscow: FIZMATLIT, 2007. [In Russian]

[4] OpenFOAM Foundation. [Online]. Available: http://www.openfoam.org.

[5] Kurganov A., Tadmor E. New high-resolution central schemes for nonlinear conservation laws and convection-diffusion equations. // J. Comput. Phys., vol. 160, pp. 241-282, 2000. 
[6] Greenshields C.J., Weller H.G., Gasparini L., Reese J.M. Implementation of semi-discrete, non-staggered central schemes in a colocated, polyhedral, finite volume framework, for high-speed viscous flows. // Int. J. Numer. Meth. Fluids, vol. 63, no. 1, pp. 1-21, 2010.

[7] Issa R. Solution of the implicit discretized fluid flow equations by operator splitting. // J. Comput. Phys., vol. 62 , no. 1, pp. 40-65, 1986.

[8] Kraposhin M., Bovtrikova A., Strijhak S. Adaptation of Kurganov-Tadmor numerical scheme for applying in combination with the PISO method in numerical simulation of flows in a wide range of Mach numbers. // Procedia Computer Science, vol. 66, pp. 43-52, 2015.

[9] Babenko K.I., Voskresenskii G.P., Lyubimov A.N., Rusanov V.V. Prostranstvennoe obtekanie gladkikh tel ideal'nym gazom, [Three-dimensional ideal gas flow past smooth bodies]. - Moscow: Nauka, 1964. [In Russian]

[10] Karvatskii A.Ya., Pulinets I.V., Lazarev T.V., Pedchenko A.Yu. Chislennoe modelirovanie sverkhzvukovogo obtekaniya klina s primeneniem svobodnogo otkrytogo programmnogo koda. [Numerical modelling of supersonic flow around a wedge with the use of free open software code OpenFOAM]. - Space science and technology, vol. 21, no. 2, pp. 47-52, 2015. [In Russian]

[11] Gutierrez L.F., Tamagno J.P., Elaskar S.A. High speed flow simulation using OpenFOAM. // Mecanica Computacional, vol. XXXI, Salta, Argentina, pp. 2939-2959, 2012.

[12] Lorenzon D., Elaskar S.A. Simulacion de flujos supersonicos bidimensionales y axialmente simetricos con OpenFOAM. // Revista de la Facultad de Ciencias Exactas, Fisicas y Naturales, vol. 2, no. 2, pp. 65-76, 2015.

\section{About the authors}

Bondarev Alexander E., PhD, senior researcher, Keldysh Institute of Applied Mathematics RAS. E-mail: bond@keldysh.ru.

Kuvshinnikov Artem E., junior researcher, Keldysh Institute of Applied Mathematics RAS. E-mail: kuvsh90@yandex.com 